\title{
The Overlap Between GERD and Functional Bowel Disorders - When East Meets Rome
}

\author{
Tiberiu Hershcovici, MD and Ronnie Fass, MD* \\ Neuroenteric Clinical Research Group, Southern Arizona VA Health Care System and University of Arizona Health Sciences Center, Tucson, \\ Arizona, USA
}

\section{Article: Overlap of erosive and non-erosive reflux diseases with functional gastrointestinal disorders according to Rome III criteria \\ (J Neurogastroenterol Motil 2010;16:148-156)}

Gastroesophageal reflux disease (GERD), functional dyspepsia (FD) and irritable bowel syndrome (IBS) are very common in the general population. GERD has been shown to be less common in the East as compared to the West. Furthermore, erosive esophagitis (EE) is usually milder (predominantly Los Angeles grades A and B), and GERD complications are relatively uncommon in Asia. ${ }^{1}$ However, an overall rapid increase in the incidence and prevalence of GERD in Asia has been reported in the last decade. ${ }^{2}$

Since the aforementioned disorders are very common, there is a higher likelihood that they will overlap by chance. However, recent epidemiologic studies have demonstrated that the overlap between IBS and GERD is greater than anticipated by coincidence. Studies clearly demonstrated that GERD is prevalent in IBS patients and vice versa. The reason for this close relationship between the 2 disorders remains unknown. Presently, there are 2 leading hypotheses that attempt to explain this relationship. The first suggests that IBS-like symptoms are part of the spectrum of GERD manifestations. The other suggests that IBS and GERD are 2 distinct disorders with similar underlying pathophysiology. ${ }^{3}$ Thus far, there is paucity of information about the overlap between GERD and IBS or FD in Asia.

Definitions for IBS and FD have varied over the years, primarily due to the short intervals in re-examining the criteria for these disorders by the Rome Committee for Functional Bowel Disorders. Consequently, the degree of overlap between these disorders and GERD depends on the definition used. In the Rome III classification, FD was redefined, and the term was replaced by 2 new symptom-related entities: epigastric pain syndrome (EPS) and postprandial distress syndrome (PDS). ${ }^{4}$ Furthermore, the Rome III criteria emphasize the possibility of demonstrating overlap between lower and upper gut functional disorders. However, the impact of Rome III criteria as compared to the previous Rome criteria (I or II) on the level of overlap between the different functional bowel disorders remains to be elucidated.

In this issue of the journal, Noh et al. compared the prevalence and risk factors for overlap between GERD [EE and non-

Received: April 8th, 2010 Accepted: April 9th, 2010

(c) This is an Open Access article distributed under the terms of the Creative Commons Attribution Non-Commercial License (http://creativecommons. org/licenses/by-nc/3.0) which permits unrestricted non-commercial use, distribution, and reproduction in any medium, provided the original work is properly cited.

*Correspondence: Professor Ronnie Fass, MD

Neuroenteric Clinical Research Group, Southern Arizona VA Health Care System, 3601 S. 6th Avenue (1-111-GI), Tucson, Arizona 85723-0001 USA

Tel: +1-520-792-1450 (ext. 5139), Fax: +1-520-629-4737, E-mail: Ronnie.Fass@va.gov

Financial support: None.

Conflicts of interest: None. 
erosive reflux disease (NERD)] and FD or IBS (defined by the Rome III criteria). This is the first published study evaluating the overlap between GERD and functional bowel disorders in an Asian population using the Rome III criteria. A total of 2,388 Korean subjects who underwent upper endoscopy for health-screening purposes were enrolled. Of those, $15 \%$ were found to have GERD (80\% with EE and 20\% with NERD). The prevalence of $\mathrm{FD}$ and IBS in this patient population was $8 \%$ and $10 \%$, respectively. In the NERD group, $74 \%$ fulfilled the diagnostic criteria for $\mathrm{FD}$, and EPS was more prevalent than PDS. Forty-two percent of NERD patients fulfilled the diagnostic criteria for IBS. Diarrhea-predominant IBS and the unclassifiedpattern were the most frequent overlapping IBS subtypes with NERD. The prevalence of FD (10\%) and IBS (11\%) in EE patients was similar to the prevalence of these functional bowel disorders in the reference group (subjects without reflux symptoms). Significant risk factors for NERD were high somatization score and having FD, while those for EE were male gender and current smoking.

Two characteristics of the patients included in this study are highly intriguing. First, the prevalence of EE in the study's cohort was unusually high. As mentioned above, approximately $80 \%$ of the GERD patients were found to have EE. Admittedly, most of them demonstrated mild esophageal inflammation (87\% Los Angeles grade A and 12\% grade B). Studies in Western populations have demonstrated that $\mathrm{EE}$ accounts for $30-40 \%$ of the patients with GERD. In addition, the prevalence of FD and IBS in this study was similar to previous reported studies. ${ }^{2,5}$ However, the extent of overlap between FD or IBS and NERD was exceptionally high in this study. In a previous population-based survey performed in Korea, only 27\% and 24\% of GERD patients suffered from FD and IBS (as defined by Rome II criteria), respectively. ${ }^{2}$

The findings of the study by Noh et al. raise questions about the characteristics of the study population. A possible explanation for the high overlap between NERD and IBS or FD is the definition of NERD used by the study authors. NERD was diagnosed based on typical GERD symptoms and normal upper endoscopy. The authors did not attempt to perform $\mathrm{pH}$ testing demonstrating abnormal esophageal acid exposure. This kind of definition of NERD allowed a substantial number of functional heartburn patients to enter the study. It has been previously shown that functional heartburn patients frequently demonstrate traits of functional bowel disorders more commonly than pH-positive NERD patients. ${ }^{6}$
Noh et al. found that the EPS subtype of FD overlapped with NERD more frequently than the PDS subtype. Presently, there are no other studies examining the overlap between NERD or $\mathrm{EE}$ and the different subtypes of $\mathrm{FD}$ as defined by Rome III criteria. Studies have shown that patients with overlap between FD and IBS reported more dysmotility-like symptoms. ${ }^{7,8} \mathrm{~A}$ recent study from China demonstrated that the PDS subtype of FD overlapped more frequently with IBS than the EPS subtype of FD. Presently, it is not clear if the high overlap between EPS and NERD, as was demonstrated in the current study, is more specific to the study's patient population or is a more general phenomenon.

In this study, there was a significant difference in the extent of overlap with FD and IBS between NERD and EE patients. Studies comparing the extent of overlap between the different GERD groups and functional bowel disorders have yielded conflicting results. In one study that used Rome II criteria and tried to exclude functional heartburn patients from the NERD group, the authors demonstrated that NERD patients had a significantly higher prevalence of FD and IBS as compared with $\mathrm{EE}$ patients. ${ }^{9}$ However, in another study, there was no difference in the prevalence of IBS (as defined by the Manning criteria) between NERD and EE patients. ${ }^{10}$ An analysis of 14 clinical trials (6,810 patients that used the ReQuest-Reflux questionnaire) revealed that IBS and dyspepsia-like symptoms are not more common in NERD patients as compared to those with EE. ${ }^{11}$ However, none of the aforementioned studies utilized Rome III criteria to diagnose FD and IBS. Thus, it is possible that the presence or absence of significant difference in the prevalence of IBS and FD between NERD and EE is driven by what criteria are used to define functional bowel disorders. To ensure better understanding of the extent of overlap between GERD groups and functional bowel disorders, authors should "level the playing field" by using only the Rome III criteria.

In summary, the very informative study by Noh et al. demonstrated a significantly higher overlap between NERD patients and functional bowel disorders, such as IBS and FD, as compared to EE patients and normal controls. This is the first study that utilized Rome III criteria to diagnose functional bowel disorders in the different GERD groups. The study also clearly demonstrated that having NERD increases patient's risk of having a functional bowel disorder. This finding will likely have important clinical implications in the management of NERD. For example, several recent studies have suggested that GERD patients who concomitantly suffer from a functional bowel disorder 
demonstrate a lower response rate to standard-dose PPI as compared to those without a functional bowel disorder. Lastly, this study may provide us with an essential clue as to why NERD patients are less likely to respond to antireflux treatment as compared with the other GERD groups.

\section{References}

1. Wong WM, Lam SK, Hui WM, et al. Long-term prospective follow-up of endoscopic oesophagitis in southern Chinese - prevalence and spectrum of the disease. Aliment Pharmacol Ther 2002;16: 2037-2042.

2. Lee SY, Lee KJ, Kim SJ, Cho SW. Prevalence and risk factors for overlaps between gastroesophageal reflux disease, dyspepsia, and irritable bowel syndrome: a population-based study. Digestion 2009;79: 196-201.

3. Gasiorowska A, Poh CH, Fass R. Gastroesophageal reflux disease (GERD) and irritable bowel syndrome (IBS) - is it one disease or an overlap of two disorders? Dig Dis Sci 2009;54:1829-1834.

4. Drossman D. Rome III: The functional gastrointestinal disorders. 3rd ed. McLean, VA: Degnon Associates, Inc., 2006
5. Wang A, Liao X, Xiong $\mathrm{L}$, et al. The clinical overlap between functional dyspepsia and irritable bowel syndrome based on Rome III criteria. BMC Gastroenterol 2008;8:43.

6. Shapiro M, Green C, Bautista JM, et al. Functional heartburn patients demonstrate traits of functional bowel disorder but lack a uniform increase of chemoreceptor sensitivity to acid. Am J Gastroenterol 2006;101:1084-1091.

7. Agreus L, Svardsudd K, Nyren O, Tibblin G. Irritable bowel syndrome and dyspepsia in the general population: overlap and lack of stability over time. Gastroenterology 1995;109:671-680.

8. Stanghellini V, Tosetti C, Paternico A, et al. Risk indicators of delayed gastric emptying of solids in patients with functional dyspepsia. Gastroenterology 1996;110:1036-1042.

9. Wu JC, Cheung CM, Wong VW, Sung JJ. Distinct clinical characteristics between patients with nonerosive reflux disease and those with reflux esophagitis. Clin Gastroenterol Hepatol 2007;5:690-695.

10. Nojkov B, Rubenstein JH, Adlis SA, et al. The influence of co-morbid IBS and psychological distress on outcomes and quality of life following PPI therapy in patients with gastro-oesophageal reflux disease. Aliment Pharmacol Ther 2008;27:473-482.

11. Fass R, Stanghellini V, Monnikes H. Baseline analysis of symptom spectrum in GERD clinical trial patients: result from ReQuest database [abstract]. Gastroenterology 2006;130(suppl 2):A629. 